\title{
CONFIRMACIÓN DE LA PRESENCIA DE AMBLYOMMA BRASILIENSE ARAGÃO, 1908 (ACARI: IXODIDAE) EN LA ARGENTINA
}

\author{
Welschen, N. M. ${ }^{1}$; Tarragona, e. L. ${ }^{2}$; Nava, S. ${ }^{2}$ \& Guglielmone, A. A. ${ }^{2}$
}

\begin{abstract}
RESUMEN
Amblyomma brasiliense Aragão, 1908 fue colectado sobre humanos en el noroeste argentino hace mas de 60 años. Estos hallazgos se tornaron inciertos por la ausencia de nuevos registros pese a trabajos continuos de ecología de garrapatas en el norte argentino. Recientemente, se obtuvo un ejemplar macho de $A$. brasiliense de la vegetación en la provincia de Misiones, cercanías de arroyo

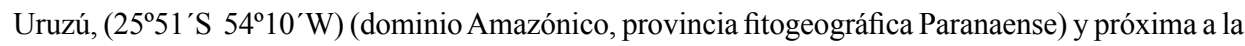
localidad de hallazgo de $A$. brasiliense en Foz de Iguaçú $\left(25^{\circ} 32^{\prime} \mathrm{S} 54^{\circ} 35^{\prime} \mathrm{W}\right)$, estado de Paraná, Brasil. El diagnóstico se obtuvo usando claves taxonómicas y por comparación con material de referencia. Se confirma la presencia de $A$. brasiliense en la Argentina, específicamente en el noreste argentino, por lo tanto sería conveniente estudios adicionales para confirmar su presencia en el noroeste argentino.

Palabras clave: Ixodidae, Amblyomma brasiliense, Dominio Amazónico, Provincia Fitogeográfica Paranaense, Argentina.
\end{abstract}

\section{SUMMARY}

Confirmation of the presence of Amblyomma brasiliense Aragão,1980 (Acari: Ixodidae) in Argentina.

Amblyomma brasiliense Aragão, 1908 has been collected from humans in northwestern Argenina more than 60 years ago. These records were becoming uncertain due to lack of new collections in spite of continuous studies on tick ecology in northern Argentina. A male of A. brasiliense was recently collected from vegetation at the vicinity of Uruzú stream $\left(25^{\circ} 51^{\prime} \mathrm{S} 54^{\circ} 10^{\prime} \mathrm{W}\right)$, province of Misiones (Amazonic domain, phytogeographic Paranaense province), which is close to the Brazilian

1.- Cátedra de Parasitología y Enfermedades Parasitarias, Facultad de Ciencias Veterinarias, Universidad Nacional del Litoral. R.P. Kreder 2805 (3080) Esperanza, Santa Fe.

2.- Instituto Nacional de Tecnología Agropecuaria, Estación Experimental Agropecuaria Rafaela, Consejo Nacional de Investigaciones Científicas y Técnicas CC 22, CP 2300 Rafaela (Santa Fe).

Manuscrito recibido el 23 de abril de 2012 y aceptado para su publicación el 14 de noviembre de 2012. 
locality of Foz de Iguaçú ( $\left.25^{\circ} 32^{\prime} \mathrm{S} 54^{\circ} 35^{\prime} \mathrm{W}\right)$, Paraná State, Brazil, where $A$. brasiliense has been also determined. The diagnosis was obtained by using taxonomic keys and comparison with reference material. This study confirms the occurrence of A. brasiliense in Argentina, specifically in northeastern Argentina, but additional efforts are needed to confirm its presence in northwestern Argentina.

Key words: Ixodidae, Amblyomma brasiliense, Amazonic Domain, Phytogeographic Paranense Province, Argentina.

\section{INTRODUCCIÓN}

Amblyomma brasiliense Aragão, 1908 es una garrapata de hallazgo relativamente frecuente en Brasil donde fue determinada para los estados de Amapá, Amazonas, Espirito Santo, Minas Gerais, Pará, Paraná, Rio de Janeiro, Rio Grande do Sul, Santa Catarina y São Paulo. Fuera de Brasil los hallazgos son esporádicos y en algunos casos controversiales. Así un registro de larvas de A. brasiliense en Bolivia por Squire (1972) es considerado dudoso en Guglielmone et al. (2003), por otra parte su presencia en Paraguay es avalada por antiguos registros en el departamento Central (Barbará y Dios, 1918) y otro en el departamento San Pedro de una garrapata colectada en 1913 (Keirans, 1985).

La situación en la Argentina es similar a la del Paraguay con un registro de una hembra de A. brasiliense parasitando un humano en San Pedro (Jujuy) en 1926 (Dios y Knopoff 1930, 1934). Boero (1945, 1955, 1957) señala su presencia en humanos en Salta sin mencionar localidad. Finalmente, Boero (1959) presenta un registro para la localidad de Media Luna (Salta) pero no menciona ningún hospedador. (Guglielmone \& Nava 2006) resumieron la situación de A. brasiliense en la Argentina indicando su parasitismo en humanos en dos localidades, una de Salta y otra de Jujuy en la provincia fitogeografía de las Yungas del dominio Amazónico.
Desde 1976 hasta el presente se desarrollaron numerosos estudios sobre garrapatas en el noroeste argentino sin que la presencia de A. brasiliense se confirmara. A ello se agrega que la iconografía para A. brasiliense en Dios y Knopoff $(1930,1934)$ arroja ciertas dudas acerca del diagnóstico específico y el material utilizado para su diagnóstico no está disponible. El presente estudio confirma la presencia de $A$. brasiliense en la Argentina pero por un espécimen obtenido en el noreste argentino.

\section{MATERIALES Y MÉTODOS}

Durante trabajos de rutina el 27 de agosto de 2012 uno de los autores (NMW) colectó un macho de garrapata del género $\mathrm{Ambl}$ yomma de la vegetación en las cercanías del arroyo Uruzú y de la ruta provincial 19 (25 $\left.51^{\prime} \mathrm{S} 54^{\circ} 10^{\prime} \mathrm{W}\right)$, departamento General Belgrano, provincia de Misiones, sitio que corresponde al dominio Amazónico en la provincia fitogeografía Paranaense de acuerdo con Cabrera (1976).

La garrapata fue clasificada con las claves de Jones et al. (1972), Guglielmone \& Viñabal (1994) y Onofrio et al. (2006). Adicionalmente, se comparó con material de referencia depositado en la colección de garrapatas del Instituto Nacional de Tecnología Agropecuaria, Estación Experimental Agropecuaria Rafaela. 